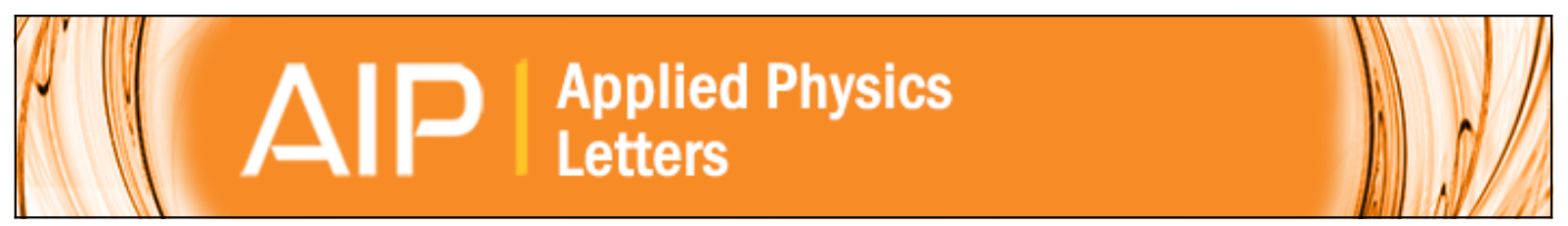

\title{
Response to "Comment on "Threshold switching via electric field induced crystallization in phase change memory devices'” [Appl. Phys. Lett. 102, 236101 (2012)]
}

J. A. Vazquez Diosdado, P. Ashwin, K. I. Kohary, and C. D. Wright

Citation: Applied Physics Letters 102, 236102 (2013); doi: 10.1063/1.4809722

View online: http://dx.doi.org/10.1063/1.4809722

View Table of Contents: http://scitation.aip.org/content/aip/journal/apl/102/23?ver=pdfcov

Published by the AIP Publishing

\section{Articles you may be interested in}

Comment on "Threshold switching via electric field induced crystallization in phase change memory devices" [Appl. Phys. Lett. 100, 253105 (2012)]

Appl. Phys. Lett. 102, 236101 (2013); 10.1063/1.4809720

Field-dependent activation energy of nucleation and switching in phase change memory Appl. Phys. Lett. 100, 263501 (2012); 10.1063/1.4731289

Threshold switching via electric field induced crystallization in phase-change memory devices Appl. Phys. Lett. 100, 253105 (2012); 10.1063/1.4729551

Electric field induced crystallization in phase-change materials for memory applications Appl. Phys. Lett. 98, 223102 (2011); 10.1063/1.3595408

Transient phase change effect during the crystallization process in phase change memory devices Appl. Phys. Lett. 94, 243504 (2009); 10.1063/1.3155200

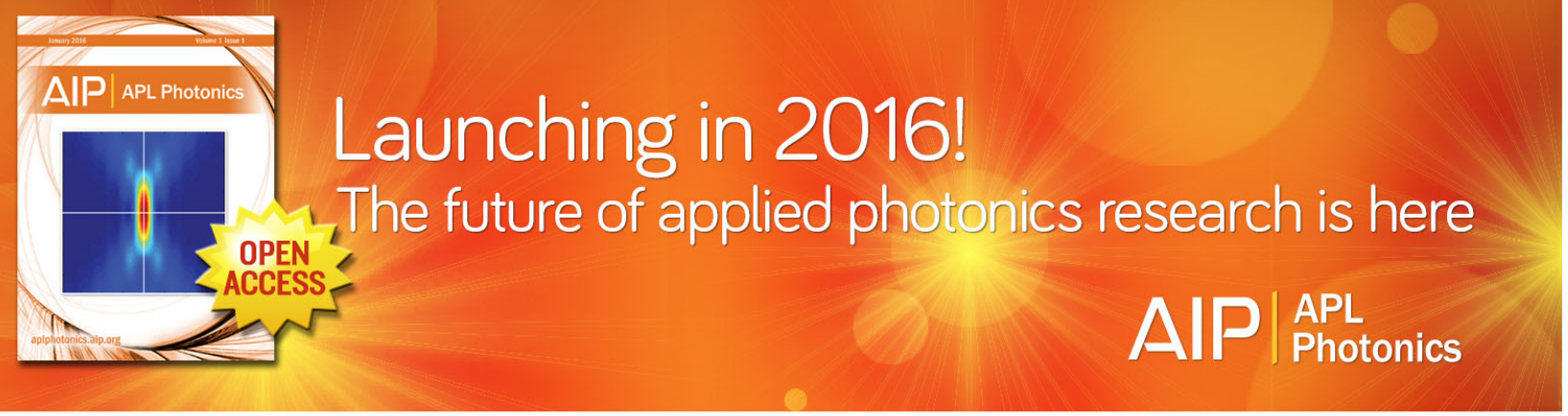




\title{
Response to "Comment on "Threshold switching via electric field induced crystallization in phase change memory devices'” [Appl. Phys. Lett. 102, 236101 (2012)]
}

\author{
J. A. Vazquez Diosdado, P. Ashwin, K. I. Kohary, and C. D. Wright \\ College of Engineering, Mathematics and Physical Sciences, University of Exeter, Exeter EX4 4QF, \\ United Kingdom
}

(Received 28 March 2013; accepted 20 May 2013; published online 10 June 2013)

[http://dx.doi.org/10.1063/1.4809722]

We find the comments of Karpov et al. regarding our paper rather surprising in several respects, since:

(i) Our paper ${ }^{1}$ showed that a PCM device switching model incorporating electric field induced crystallization, but excluding any electronic effects, predicted electrical I-V responses of the form seen in real devices, so providing some support for the possibility that electric field induced crystallization plays a role in threshold switching.

(ii) The key parameter in the energy term for electric field induced crystallization is the ratio of permittivity $(\varepsilon)$ to depolarizing factor (n); in our paper we used $\varepsilon / \mathrm{n}=300$ which is in fact suitable for modeling elongated particles for the permittivity value chosen by Karpov et al. (and in any case, the role of varying the depolarizing factor was specifically examined in works published by us both before ${ }^{2}$ and after $^{3}$ the paper to which the current comments are addressed).

(iii) The crystallization models used in our work are based on standard and well-accepted approaches, namely, classical nucleation theory and a rate-equation based growth/dissociation model. ${ }^{4}$

We now expand on the points (i)-(iii) and make pertinent responses to more general comments from Karpov et al. regarding our simulation approach.

To model the possible effects of field induced nucleation, our paper included the additional, as compared to conventional crystallization models, electric field-related free energy term, $g(E)$, which is identical in form to that used by Karpov et al., i.e., $g(E)=-0.5 E^{2} \varepsilon_{0} \varepsilon / n$ (in SI units), where $E$ is the electric field, $\varepsilon$ the (relative) permittivity, and $n$ the depolarizing factor (for a nucleated crystalline particle). For $\varepsilon / n=300$, the value in our paper, and $\varepsilon=10$, the permittivity value used by Karpov et al., this leads to a depolarizing factor of $n=1 / 30$, equivalent to that for an elongated particle such as a prolate ellipsoid with major/minor axis ratio greater than 14 , or an elongated cylinder with height/radius ratio of around 7 (or $\sim 5.5$ using the $\mathrm{n} \sim(\mathrm{R} / \mathrm{H})^{2}$ approximation), as shown in Fig. 1. Alternatively, if a permittivity value of 100 is assumed, as in our paper, then an $\varepsilon / n$ ratio of 300 is equivalent to that for a spherical particle. However, the key point is that the electric field induced energy term $g(E)$ is dependent on the ratio of $\varepsilon / n$, and how this is interpreted in terms of the types of particles it represents is immaterial. That is not to say that the choice of parameter values is un-important, far from it, since the choice of appropriate parameter values is crucial to the validity of modeling and simulation results.

In our modeling work, we always choose parameter values based on relevant experimental measurements or on "accepted" literature values, where available. For example, important parameters in classical nucleation theory are the surface energy $\sigma$ (here the interfacial energy density between amorphous and crystalline phases) and the bulk free energy difference (chemical potential) $\Delta g$ between the two phases, which is usually described in terms of the enthalpy of fusion $\mu_{f}$ along the lines of $\Delta g=\mu_{f}\left[1-T / T_{m}\right]$ where $T_{m}$ is the melting temperature. In our work we chose $\sigma=0.066 \mathrm{~J} \mathrm{~m}^{-2}$ and $\mu_{f}=1121 \mathrm{~J} \mathrm{~cm}^{-3}$, values typical of those that have featured extensively in the literature. ${ }^{4-9}$ Karpov et al. in their calculations, on the other hand, choose parameters that lead to much lower values for such surface energy and enthalpy terms. Specifically, in their comment, a nucleation barrier, $W_{0}$, of $1 \mathrm{eV}$ is chosen, along with a critical radius, $R_{0}$, of $2 \mathrm{~nm}$; this leads, for spherical particles, to a very low surface energy term (given simply by $\sigma=\mathrm{W}_{0} /\left[(4 \pi / 3) \cdot \mathrm{R}_{0}{ }^{2}\right]$ ) of $0.01 \mathrm{~J} \mathrm{~m}^{-2}$, as well as an extremely low enthalpy term (given very approximately by $2 \sigma / \mathrm{R}_{0}$ ) of the order $10 \mathrm{~J} \mathrm{~cm}^{-3}$. Clearly, the choice of parameter values can and will have a significant effect on predictions for theoretical models and resulting computer simulations. We illustrate this in the inset of Fig. 1 where we

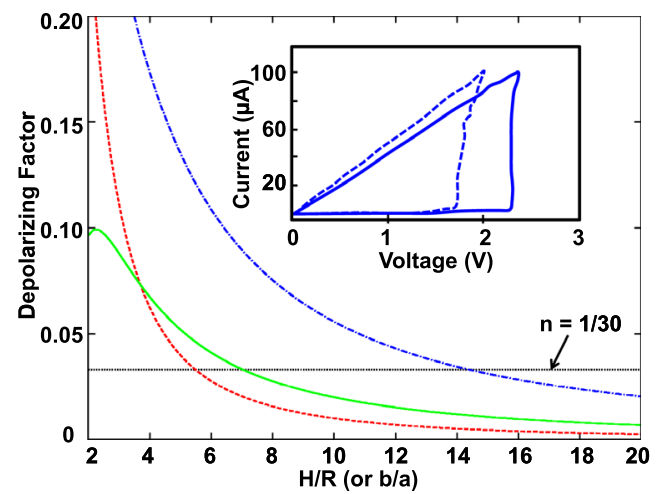

FIG. 1. Calculated depolarizing factors for a prolate ellipsoid as a function of b/a (dashed-dotted line) and for a cylinder with height/radius ratio $\mathrm{H} / \mathrm{R}$ for (i) the specific case $n=(R / H)^{2}$ (dotted line) and (ii) a more general approximation (solid line, see supplementary material of Ref. 1). Particle elongation (length/width ratio) for the case corresponding to $\varepsilon / n=300$ with $\varepsilon=10$ can be read off from the intersection of the horizontal line shown with the appropriate curve. Inset shows simulated PCM I-V curves as in our original paper (solid line) and for an increased $\varepsilon / n$ ratio of 1000 (dotted line). 
show the predicted I-V curve for the PCM "mushroom-cell" device of our original paper for the case when $\varepsilon / n=1000$ (equivalent to a depolarizing factor of 0.01 for $\varepsilon=10$ ).

Karpov et al. also comment on the potential limitations of the use of $2 \mathrm{D}$ modeling. Essentially our models are pseudo-3D, since the electro-thermal equations are solved in cylindrical co-ordinates for the cylindrically symmetric PCM "mushroom" cell. Furthermore, in our rate-equation driven phase-change model, the 3D surface area and volume of a monomer is used in calculating the energy associated with crystal nucleation and growth events. ${ }^{10}$ Moreover, we note that we have, in previous work, compared the results of full-3D electro-thermal and phase-change simulations to those of the 2D approach and found that both predict very similar features and effects. ${ }^{11}$ With regard to the comment that our approach uses a "succession of elemental site transformations" to represent nucleation, we note that while the Gillespie algorithm ${ }^{12}$ assumes that reactions (in this case dimer formation and subsequent growth and dissociation events) occur in succession with infinite speed, this is a standard computational approach used for simulation of chemical reactions and we believe it is appropriate here. As long as the time-step chosen for the thermal/electrical update is small enough to resolve the changes due to the change in electric field resulting from a change in phase, results will not be limited by any "conceptual flaw." We have compared our Gillespie-based cellular automata approach and the rate and master equation descriptions on which it is based, to each other and to experiment, and found good agreement. ${ }^{4,10,13}$

Finally, we reiterate that, in our view, our work provides useful evidence that field induced nucleation can lead to I-V curves in PCM devices of the form observed experimentally. Using material parameter values that we believe to be appropriate, the threshold fields (hence switching voltages) for field induced nucleation were predicted to be higher than those predicted by electronic switching models. ${ }^{14}$ However, universally accepted parameter sets for phase-change materials, even the ubiquitous $\mathrm{Ge}_{2} \mathrm{Sb}_{2} \mathrm{Te}_{5}$ alloy, are not available and, as we showed recently, ${ }^{15}$ crystallization behavior can change dramatically for relatively small changes to parameter values. Thus, as pointed out by Karpov et al. in their comment, further experimental evidence and informed debate in the scientific community is needed to determine the precise nature of threshold-switching in phase-change materials and fully understand, to quote a recent work by Hudgens, ${ }^{16}$ "the 'deceptively simple' two terminal devices that Stan Ovshinsky described 44 years ago (and) still provide us with a fascinating mystery."

${ }^{1}$ J. A. V. Diosdado, P. Ashwin, K. I. Kohary, and C. D. Wright, Appl. Phys. Lett. 100, 253105 (2012).

${ }^{2}$ K. Kohary and C. D. Wright, Appl. Phys. Lett. 98, 223102 (2011).

${ }^{3}$ K. Kohary, J. A. V. Diosdado, P. Ashwin, and C. D. Wright, Phys. Status Solidi B 249, 1897 (2012).

${ }^{4}$ S. Senkader and C. D. Wright, J. Appl. Phys. 95, 504 (2004).

${ }^{5}$ D.-H. Kim, F. Merget, M. Forst, and H. Kurz, J. Appl. Phys. 101, 064512 (2007).

${ }^{6}$ C. Peng, L. Cheng, and M. Mansuripur, J. Appl. Phys. 82, 4183 (1997).

${ }^{7}$ A. Redaelli, A. Pirovano, A. Benvenuti, and A. L. Lacaita, J. Appl. Phys. 103, 111101 (2008).

${ }^{8}$ B. Hyot, V. Gehanno, B. Rolland, A. Fargeix, C. Vannufel, F. Charlet, B. Bechevet, J. M. Bruneau, and P. J. Derse, J. Magn. Soc. Jpn. 25, 414 (2001).

${ }^{9}$ K. Sonoda, A. Sakai, M. Moniwa, K. Ishikawa, O. Tsuchiya, and T. Inoue, IEEE Trans. Electron Devices 55, 1672 (2008).

${ }^{10}$ P. Ashwin, B. S. V. Patnaik, and C. D. Wright, J. Appl. Phys. 104, 084901 (2008).

${ }^{11}$ L. Wang, Ph.D. dissertation, University of Exeter, 2009.

${ }^{12}$ D. T. Gillespie, J. Phys. Chem. 81, 2340 (1977).

${ }^{13}$ K. B. Blyuss, P. Ashwin, A. P. Bassom, and C. D. Wright, Phys. Rev. E 72, 011607 (2005).

${ }^{14}$ D. Ielmini and Y. Zhang, J. Appl. Phys. 102, 054517 (2007).

${ }^{15}$ K. Kohary and C. D. Wright, Phys. Status Solidi B 250, 944 (2013).

${ }^{16}$ S. Hudgens, Phys. Status Solidi B 249, 1951 (2012). 